\title{
Efficacy of Interferon- $\beta$ in Moderate-to-Severe Hospitalised Cases of COVID-19: A Systematic Review and Meta-analysis
}

\author{
Subodh Kumar ${ }^{1} \cdot$ Manoj Kumar Saurabh ${ }^{1} \cdot$ Venkata Lakshmi Narasimha $^{1} \cdot$ Vikas Maharshi $^{1}$ (C)
}

Accepted: 6 October 2021 / Published online: 23 October 2021

(C) The Author(s), under exclusive licence to Springer Nature Switzerland AG 2021

\begin{abstract}
Background and Objective Interferon- $\beta$, as with several other anti-viral agents, has been investigated as a treatment option for COVID-19 as a repurposed drug. The present study is a systematic review and meta-analysis of interferon- $\beta$ to determine its efficacy among moderate-to-severe COVID-19 patients.

Methods A systematic literature search was done using relevant terms for 'COVID-19' and 'interferon- $\beta$ '. Randomised controlled trials (RCT) evaluating the efficacy of interferon- $\beta$ in COVID-19 were included. Data were extracted for outcome measures, namely mortality, time to clinical improvement and length of hospital stay. Random effects meta-analysis was performed using RevMan V.5.4.1 to calculate overall effect estimate as odds ratio/hazard ratio for categorical variables and mean difference for continuous variable.

Result Eight RCTs were eligible for qualitative synthesis and seven for meta-analysis. The overall effect estimate (odds ratio $[\mathrm{OR}]$ 0.59; $95 \% \mathrm{CI} 0.91,1.12$ ) and (mean difference $[\mathrm{MD}]-1.41 ; 95 \% \mathrm{CI}-2.84,0.02$ ) indicated no statistically significant difference between effect of IFN- $\beta$ and that of control on mortality and length of hospital stay, respectively. However, the overall effect estimate (hazard ratio [HR] $1.95 ; 95 \%$ CI 1.36, 2.79) denoted a favourable effect of INF- $\beta$ on reducing the time to clinical improvement in moderate-to-severe COVID-19 patients.

Conclusion Addition of interferon- $\beta$ to standard of care resulted in significant reduction in time to clinical improvement but no significant benefit in terms of reduction in mortality and length of hospital stay in moderate-to-severe cases of COVID-19.
\end{abstract}

\section{Key Points}

Many anti-viral agents, including interferon- $\beta$, have been investigated for the treatment of COVID-19.

There are limited data on the effect of interferon- $\beta$ on outcome of COVID-19 in moderate-to-severe cases.

This systematic review and meta-analysis included all the available randomised clinical trials and evaluated the effect of interferon- $\beta$ on outcome of COVID-19 in moderate-to-severe cases.

Interferon- $\beta$ significantly reduced the time to clinical improvement but did not affect mortality and length of hospital stay outcomes as compared to standard of care.

Vikas Maharshi

vikas.maharshi81@gmail.com

1 All India Institute of Medical Sciences, Deoghar, India

\section{Introduction}

Severe acute respiratory syndrome Coronavirus 2 (SARSCoV-2), the causative factor for Coronavirus disease 2019 (COVID-19), like SARS-CoV and Middle-East respiratory syndrome coronavirus (MERS-CoV), is a member of the beta Coronaviridae family of Coronaviruses [1]. COVID-19 pandemic has affected approximately 235 million individuals, resulting in more than four million fatalities, according to the World Health Organization (WHO) [2]. Symptoms of the disease may range from fever, cough, sore throat and rhinorrhoea to severe pneumonia and septic shock. A few patients develop acute respiratory distress syndrome (ARDS), which leads to admission to the intensive care unit (ICU) and possible mortality in a significant proportion of patients $[3,4]$. Despite great efforts across the world to find potential therapeutic agents, a handful of the medicines have been approved for emergency use, and none has been accepted globally as definitive treatment of this disease. Therefore, search for effective pharmacotherapy against COVID-19 is still ongoing. Literature suggests that COVID-19 severity may correlate with 
interferon-beta (IFN- $\beta$ ) levels in the body [5]. Interferons are well known for their antiviral as well as immunomodulatory properties. These are classified as type I, II and III IFNs. Type I IFNs are IFN- $\alpha$, IFN- $\beta$, IFN- $\omega$, IFN- $\kappa$ and IFN- $\tau$ [6-8]. Type I IFNs have been found to reduce SARS-CoV-2 replication in Vero E6 cells in vitro assay, viral antigen expression, viral load reduction and plaque reduction assays [9]. Furthermore, IFN$\beta 1 \mathrm{~b}$ has been shown to have potential to reduce virus-induced lung fibrosis in a mouse model, which could be advantageous for COVID-19 patients with ARDS [10]. It has been found that SARS-CoV-2 could prevent IFN production by such mechanisms as escaping from recognition by pattern recognition receptors $[11,12]$, interfering with retinoic acid-inducible gene I or toll-like receptor signalling [13] and inhibiting phosphorylation of interferon regulatory factor 3 and its activation [14]. Because of its antiviral and immunomodulatory properties, IFN- $\beta$ has been and is being investigated for management of COVID-19, especially the severe form of the disease.

Variable results of treatment with IFN on COVID-19 have been found in clinical trials; therefore, a systematic review and meta-analysis was planned. The objectives of this systematic review and meta-analysis are to assess the effect(s) of IFN- $\beta$ therapy in moderate-to-severe cases of COVID-19 in terms of mortality, length of hospital stay (LOHS) and time to clinical improvement, to help make decisions regarding selection of this drug as a therapeutic option for treatment of this disease.

\section{Materials and Methods}

\subsection{Literature Search Strategy}

Literature search was done in PubMed/MedLine, Google Scholar, EMBASE and SCOPUS. PubMed search was performed using terms namely 'Coronavirus Infection', 'COVID-19', 'SARS-CoV-2', 'severe acute respiratory syndrome coronavirus 2 ' in combination with 'Interferon beta', 'Interferon $\beta$ ' and 'IFN- $\beta$ '. Search in Google scholar, EMBASE and SCOPUS was made using keyword 'Interferon beta for COVID-19'. Last search was made on July 31, 2021. This review was registered with PROSPERO (registration number CRD42021262804).

\subsection{Inclusion/Exclusion Criteria}

Randomised controlled trials (published up to July 2021) comparing efficacy of IFN- $\beta$ to that of standard of care in terms of mortality, LOHS, and/or time to clinical improvement, were included. Studies with significant inconsistency between experimental and control groups and studies with use of IFN-beta in experimental as well as in control study arms, were excluded from meta-analysis (Table 1).

\subsection{Data Extraction and Quality Assessment}

Two reviewers screened the search results with titles and abstracts to find eligible studies. Then the full text of initially screened studies was accessed and data from intervention and control group were recorded in Microsoft Excel. The main data fields were authors, year of publication, trial identifier, study design, population, sample size, intervention(s), comparator(s), dosage and outcome(s) measured. The quality of each study was evaluated independently by 'Cochrane Risk of Bias assessment tool version 2' (RoB 2), which had the facility to assess for randomisation, allocation concealment, blinding, completeness and other sources of bias(es). The response options for each risk of bias judgement were low risk, some concern and high risk of bias.

\subsection{Outcome Indicators}

The eligible studies included hospitalised patients with moderate-to-severe COVID-19. The outcome parameters of this systematic review and meta-analysis were: (1) hospital mortality, measured in terms of proportion of participants dying between days 14-28 days (duration of study), (2) LOHS, number of days spent by participants in the hospital from the day of admission up to the day of their discharge and (3) time to clinical improvement, defined as time in terms of days required for improvement of score at least by ' 2 ' points on an ordinal scale, namely National Early Warning Score 2 (NEWS2) scale [15] and/or WHO clinical progression scale [16].

\subsection{Statistical Analysis}

Data were entered into the Microsoft Excel. Meta-analyses were done using the software 'Review Manager (RevMan)' version 5.4.1 using random effect model. Relevant summary measures of efficacy were assessed using the odds ratio (OR), hazard ratio (HR) and/or mean difference (MD) for applicable variables along with corresponding $95 \%$ confidence interval (CI). Cochrane ' $Q$ ' statistic was applied for statistical heterogeneity, which was quantified using the $I^{2}$ statistic. $I^{2}$ value below 30 was considered as 'low', 30-59 as 'moderate', 60-74 as 'substantial' and $\geq 75$ as considerable heterogeneity. A $p$ value of $<0.05$ was considered as statistically significant.

\section{Results}

The initial search identified 1338 potential citations, of which 65 records were screened and the rest were excluded by looking into the titles and/or abstracts. Twenty-four 
records were found to be original research articles and were assessed for eligibility. Of these 24 studies, 16 were excluded as they were either nonclinical or non-randomised studies. The remaining eight studies [10,16-22] were included in qualitative synthesis (systematic review), but one study [22] was excluded from meta-analysis as it used IFN in experimental as well as in the control arm. All seven of the remaining articles were included in the meta-analysis (Fig. 1).

\subsection{Outcome Parameters}

\subsubsection{Mortality}

In all the seven studies included in meta-analysis, mortality was one of the study outcomes. One of the included studies [10] did not find death in any of the study arms; however, it has been retained in the analysis as mortality as an outcome was evaluated in this study. Random effect model was used for meta-analysis. Number of events was put in the software and calculated odds ratio was used for interpretation. The overall effect estimate [OR 0.59; $95 \%$ CI 0.91, 1.12] indicates no statistically significant difference between effect of IFN- $\beta$ and that of control on mortality. Overall heterogeneity was moderate with $I^{2}$ value of $57 \%$ which was statistically significant $(p=0.04)$. A sensitivity analysis, performed to exclude 'WHO SOLIDARITY trial' [21] (accounting for the larger sample size), showed a significantly lower mortality in IFN- $\beta$ intervention than that in control arm (Fig. 2A).

\subsubsection{Length of Hospital Stay}

The LOHS was one of the study outcomes in four of the seven studies. One study [20] had two experimental arms (with IFN- $\beta 1 \mathrm{a}$ and IFN- $\beta 1 \mathrm{~b}$ as two interventions, respectively), which were compared with control. The results of both these experimental arms have been included separately for meta-analysis. Random-effect model was used for analysis. The overall effect estimate, (MD - 1.41; 95 $\% \mathrm{CI}-2.84,0.02$ ) denotes a marginally favourable effect of INF- $\beta$ on decreasing the LOHS compared to that of
Fig. 1 PRISMA flow chart showing inclusion of studies in systematic review and metaanalysis

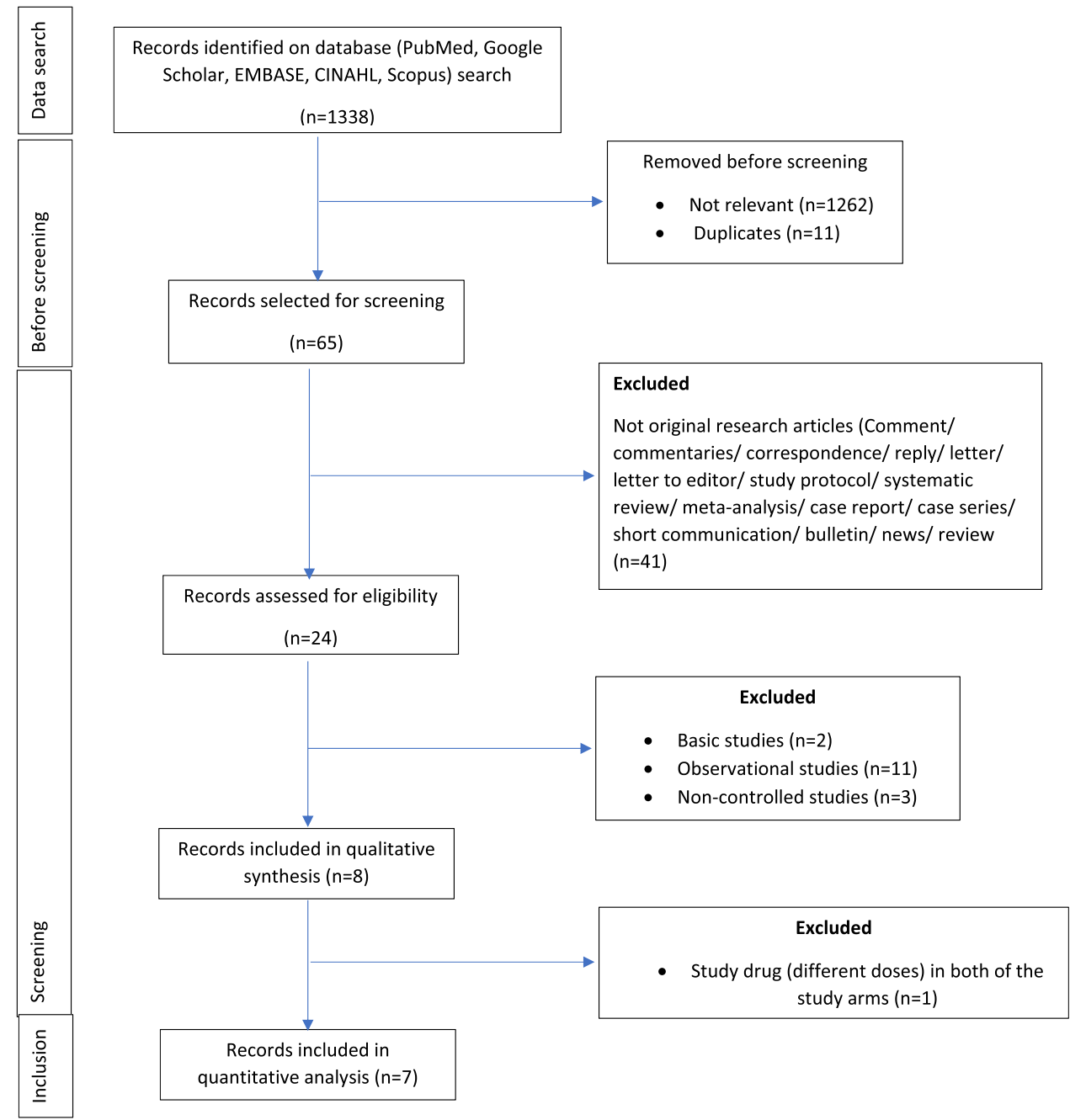


control, but effect was not statistically significantly different $(p=0.05)$ from that shown by control. Substantial heterogeneity with $I^{2}$ value of $64 \%$ was found among studies (Fig. 2B).

\subsubsection{Time to Clinical Improvement}

The data on time to clinical improvement have been reported in four of the studies. As mentioned above, results of two experimental arms (IFN- $\beta 1 \mathrm{a}$ and IFN- $\beta 1 \mathrm{~b}$ ) from one study [20] are used separately here as well, for meta-analysis. Random effect model was used during analysis. Overall effect estimate (HR 1.95; $95 \%$ CI 1.36, 2.79) shows significantly less ( $p=0.0003$ ) time to clinical improvement with IFN- $\beta$ intervention than that with the control.
Moderate heterogeneity ( $\left.I^{2} 39 \%\right)$ was found among the studies (Fig. 2C).

\subsection{Risk of Bias Assessment for Included Studies}

Of seven randomised controlled trials, two were deemed to be of 'high-risk', another two were of 'some concern' and the rest were regarded as having 'low risk' following assessment of risk of bias through Cochrane's RoB-II tool (Fig. 3).

\subsection{Publication Bias}

Funnel plot test has minimal role in cases where the number of included studies is less than 10. However, to meet the requirement of PRISMA checklist, using the inverted funnel plot method, a scatter diagram was generated with the OR on

A

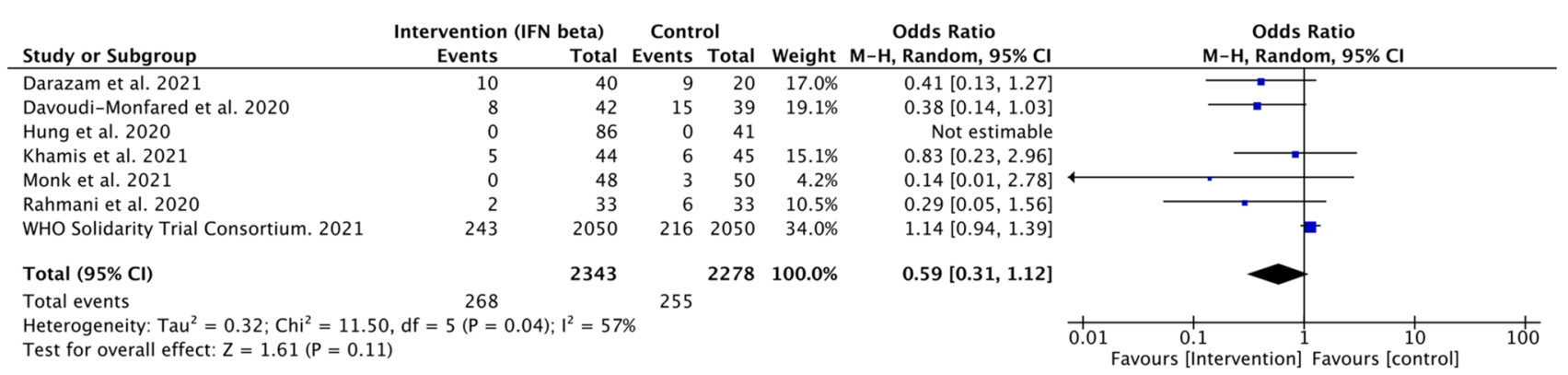

B

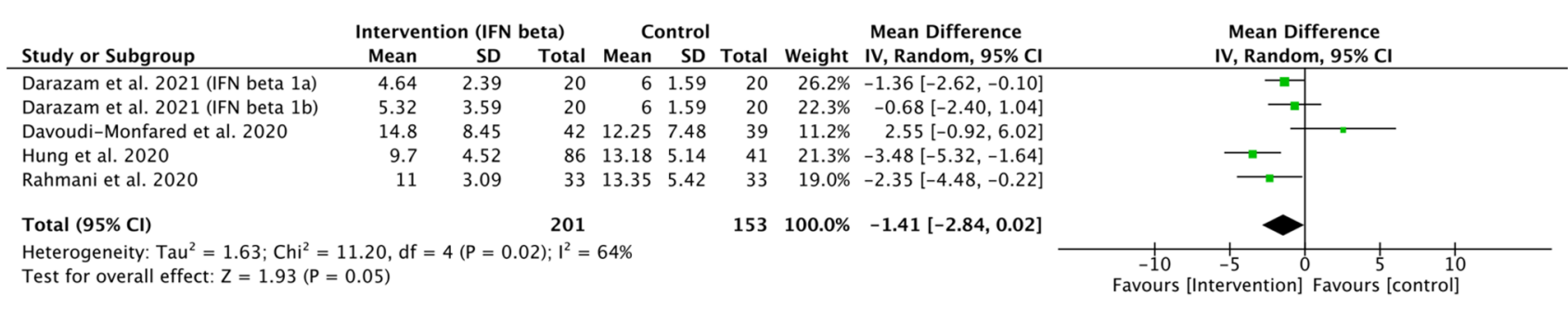

C

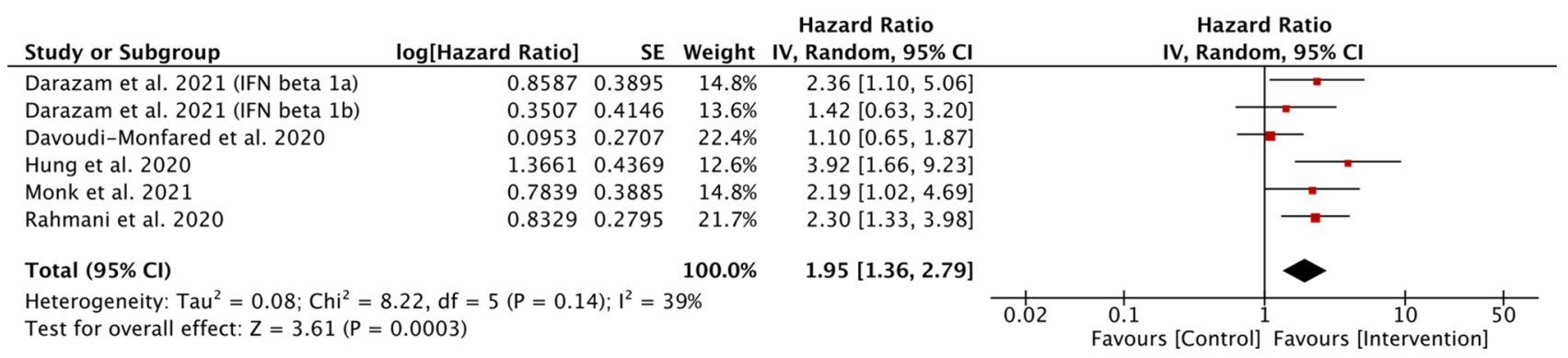

Fig. 2 Forest plots for various outcome parameters included in meta-analysis: A mortality; B length of hospital stay; $\mathbf{C}$ time to clinical improvement. $C I$ confidence interval, $I F N$ interferon, $S D$ standard deviation 
the abscissa and standard error (log [OR]) on the ordinate. Number of studies included in this meta-analysis was insufficient to distinguish a chance from real asymmetry (Fig. 4).

\section{Discussion}

IFN- $\beta$, a subtype of type I IFN, has been investigated widely as one of the treatment options for COVID-19 as a repurposed drug [11, 16-22]. The present piece of work is a systematic review and meta-analysis of the randomised controlled trials evaluating the efficacy of IFN- $\beta$ in terms of mortality, LOHS and time to clinical improvement. Although latter two parameters seem to be overlapping and measuring the same clinical outcome, there is a subtle difference between these two outcome parameters. The 'LOHS' measures the time spent by the participants in hospital (duration from the day of admission to the day of discharge) whereas 'time to clinical improvement' measures a time required for an upgradation of clinical status of participant specified by a predefined increase in the score on an appropriate ordinal scale.

A total of eight randomised control trials were included in the systematic review, of which seven were included in the meta-analysis. One study [22] was excluded from the metaanalysis as IFN- $\beta$ (although in different doses) was used in both of the study arms (Table 1).

\subsection{Mortality}

Individually, no included studies found significant impact of IFN- $\beta$ on mortality in COVID-19 patients as compared to standard of care/control. Also, pooled data in this metaanalysis found no significant effect of this intervention on mortality compared with control [OR $0.59 ; 95 \%$ CI 0.31-1.12]. However, the analysis results might have drawn largely by the WHO Solidarity trial with a larger sample size. The sensitivity analysis performed that excluded this study shows a significant reduction in mortality as compared to control group, OR, 0.45 [95\% CI 0.24-0.82]. The possible explanation for moderate heterogeneity among studies pooled to analyse data on mortality could be an outlying sample size, varying dosage used and varying characteristics of study population. The WHO Solidarity trial, which investigated the effects of four interventions in COVID-19 patients namely: remdesivir, hydroxychloroquine, lopinavir/ ritonavir and IFN- $\beta$, found no significant effect of any of the trial drugs on mortality [23], which was the chief aim of this trial. Based on interim results, the IFN- $\beta$ arm was discontinued from this trial. Another trial 'Adaptive COVID-19 Treatment Trial 3 (ACTT-3)' that evaluated the effect of IFN- $\beta$ in combination with remdesivir versus remdesivir plus placebo, has completed its recruitment (969 participants) and results are still awaited [24]. This study could be helpful in decision making regarding use of IFN- $\beta$ as a potential therapeutic agent in COVID-19. No meta-analyses were found to assess the effect of IFN- $\beta$ on mortality in COVID-19 patients. Nakhlband et al. [25] conducted a meta-analysis to evaluate the effect of IFN- $\beta$, but did not analyse for mortality.

\subsection{Length of Hospital Stay (LOHS)}

In the present meta-analysis, IFN- $\beta$ was found to reduce the LOHS but this benefit was not statistically significant as compared to that conferred by control (MD - 1.41; 95 \% CI $-2.84,0.02)$. In two of the included studies $[10,18]$ LOHS was significantly less in IFN- $\beta$ arm than that in the control arm. Overall heterogeneity among included studies was found to be moderate. Sensitivity analysis revealed that exclusion of one study (Davoudi-Monfared et al. 2020 [19]) reduced the heterogeneity to $46 \%$ with negligible change in overall LOHS. Nakhlband et al. [25] analysed the effect of IFN- $\beta$ on LOHS in their meta-analysis, which included three studies, and found a significant reduction in the LOHS by IFN- $\beta$ in comparison to control. Another systematic review by Sosa et al. [26] also shows a decrease in overall hospital stay following addition of IFN- $\beta$ to the current standard of care. However, WHO Solidarity trial found no significant difference in LOHS between groups of COVID-19 patients treated with IFN- $\beta$ compared to standard of care [21, 23]. Results of this meta-analysis may be considered in line with the findings of WHO Solidarity trial because the benefit found is only marginal and was not statistically significant.

\subsection{Time to Clinical Improvement}

Four studies in the literature were found that showed significantly less time to clinical improvement with IFN- $\beta$ as compared to that with control $[10,17,18,20]$. Similarly, pooled data from the included studies in this meta-analysis show significantly less $(p=0.0003)$ time to clinical improvement in COVID-19 patients receiving IFN- $\beta$ compared to those receiving standard of care. The test for heterogeneity shows $I^{2}$ value of $39 \%$, which is in the moderate category.

One study by Darazam et al. [20, 22] further evaluated low-dose (three doses of 12 million IU) versus high-dose (three doses of 24 million IU) IFN- $\beta 1$ a in hospitalised patients with moderate-to-severe COVID-19. This study found no significant difference $(p=0.55)$ in mortality between the two study arms. Interestingly, time to clinical improvement was significantly shorter $(p=0.018)$ in the low-dose arm as compared to that in the high-dose arm [22]. Longer time to clinical improvement in case of high-dose IFN- $\beta$ may be due to more adverse events in this arm.

In the included studies, IFN- $\beta$ was administered irrespective of magnitude of predicted IFN response. It is worth noting that only a subpopulation of COVID-19 
A

\section{As percentage (intention-to-treat)}

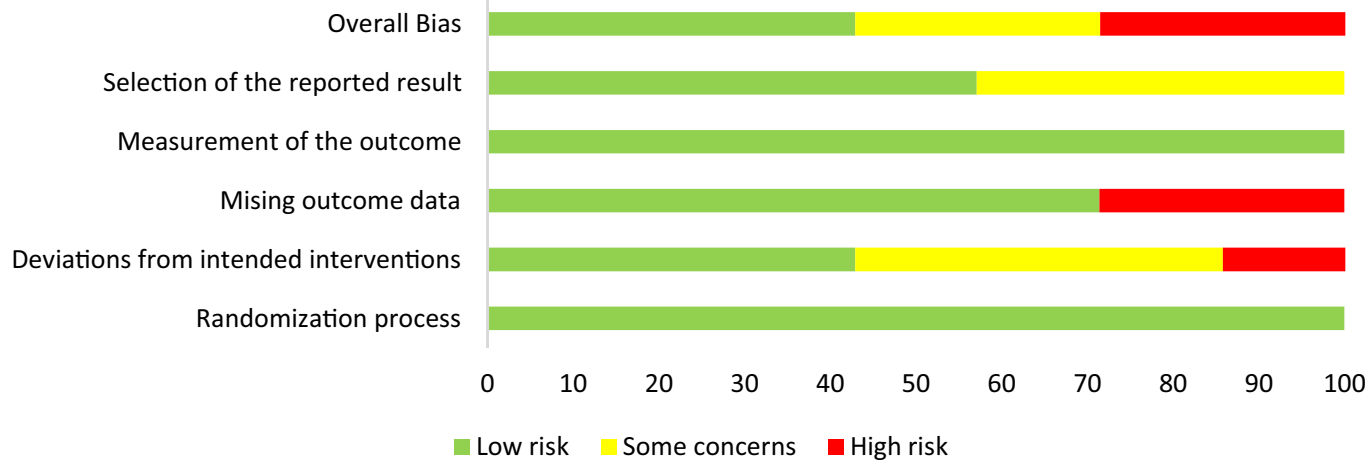

B

\begin{tabular}{|c|c|c|c|c|c|c|c|}
\hline & Study ID & D1 & D2 & D3 & D4 & $\underline{\text { D5 }}$ & Overall \\
\hline \multicolumn{8}{|c|}{ Rahmani et al. 2020} \\
\hline \multicolumn{8}{|c|}{$\begin{array}{l}\text { Davoudi-Monfared et } \\
\text { al. } 2020\end{array}$} \\
\hline \multicolumn{8}{|c|}{ Khamis et al. 2021} \\
\hline \multicolumn{8}{|c|}{ Darazam et al. 2021} \\
\hline \multicolumn{8}{|c|}{$\begin{array}{l}\text { WHO Solidarity Trial } \\
\text { Consortium. } 2021\end{array}$} \\
\hline \multicolumn{8}{|c|}{ Hung et al. 2020} \\
\hline \multicolumn{8}{|c|}{ Monk et al. 2021} \\
\hline D1 & \multicolumn{7}{|c|}{ Randomisation process } \\
\hline $\mathrm{D} 2$ & \multicolumn{7}{|c|}{ Deviations from the intended interventions } \\
\hline D3 & \multicolumn{7}{|c|}{ Missing outcome data } \\
\hline D4 & \multicolumn{7}{|c|}{ Measurement of the outcome } \\
\hline D5 & \multicolumn{7}{|c|}{ Selection of the reported result } \\
\hline
\end{tabular}

Fig. 3 Traffic light plot for risk of bias assessment through Cochrane RoB2 tool: A summary diagram; B percentile chart. + indicates low risk; (?indicates some concern; - indicates high risk 
Fig. 4 Funnel plot of publication bias of randomised controlled trials included in meta-analysis. Six studies are shown because one study [10] had number of events as ' 0 '; however, because mortality was assessed as one of the outcome parameters, the study was included in the meta-analysis. $O R$ odds ratio, SE standard error

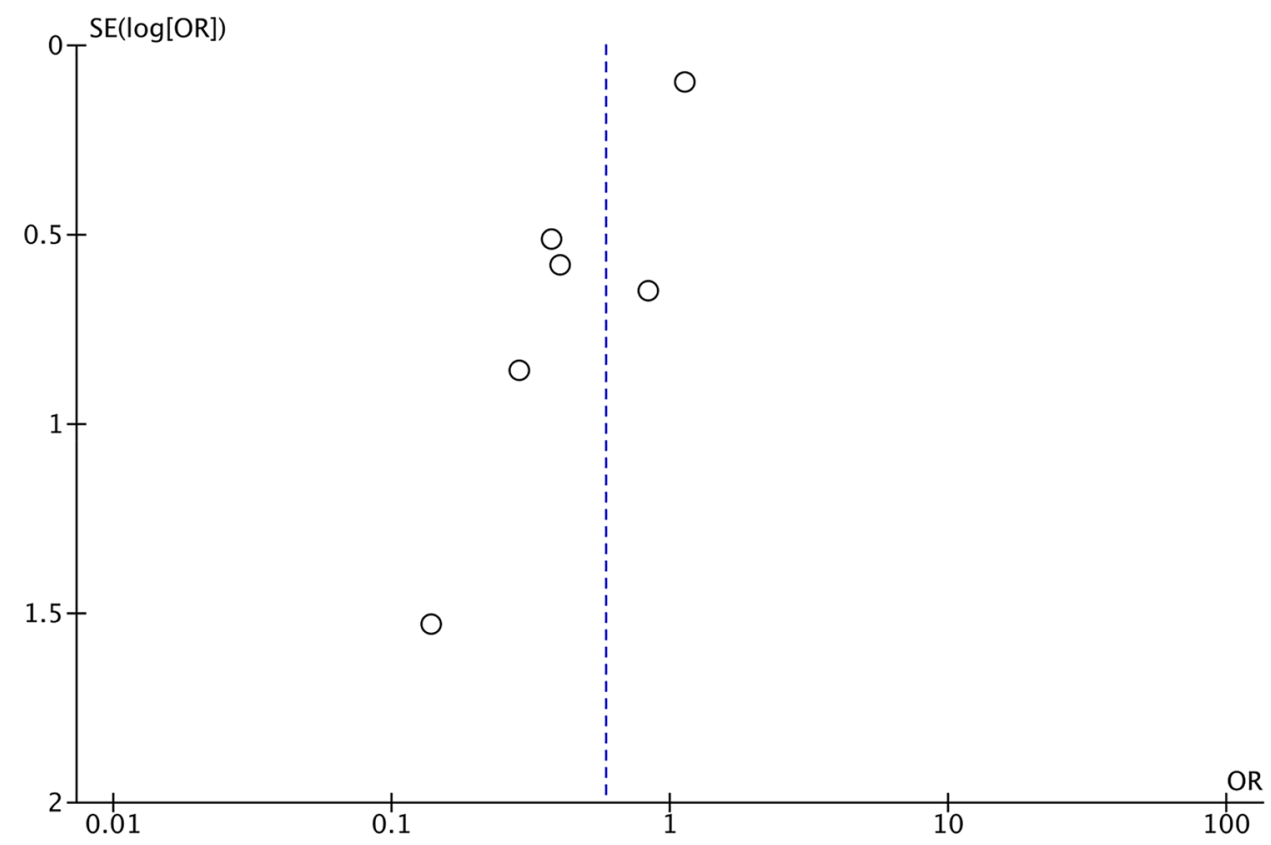

patients suffers from a defective type I IFN response, while some others produce stronger response [27]. Indeed, screening patients for IFN impairment, by quantifying IFN- $\beta$ levels with highly sensitive assays and/or interferon stimulated gene expression by real time-polymerase chain reaction technique could have been performed on the day of admission to determine which patients may benefit from IFN treatment. Moreover, the timing of IFN- $\beta$ administration is also important as administration in the early stage of the infection may result in a favourable clinical outcome [19]. In this regard, the number of properly designed studies and those designed to investigate the early versus late effect of IFN- $\beta$ in COVID-19 patients is low, and future studies are needed to further confirm the importance of IFN- $\beta$ therapy in COVID-19 taking these factors into consideration.

\section{Conclusion}

This systematic review and meta-analysis found no significant benefit of IFN- $\beta$ in terms of reduction in mortality and LOHS among COVID-19 patients. The possible reasons for the same could be the limited number of the eligible studies, different dosage of IFN $-\beta$ and presence of confounding factors like concomitant pharmacotherapy. Moreover, due to the heterogeneity of the disease between individuals, possibly due to different factors such as genetics, age and sex, the response to anti-COVID-19 treatment may be variable. However, this study favours use of IFN- $\beta$ in terms of reducing the time to clinical improvement in moderate-to-severe COVID-19 patients. 


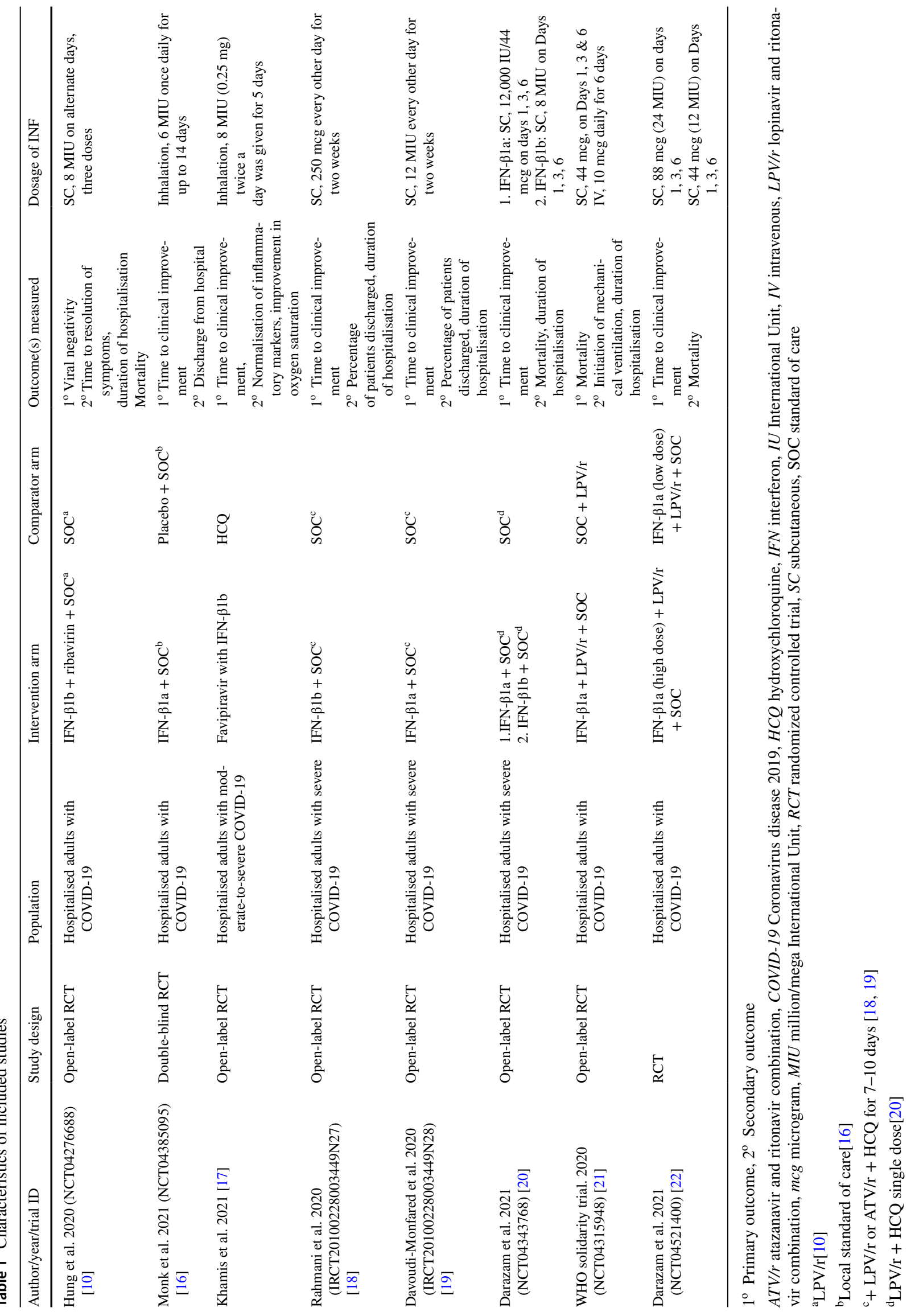




\section{Declarations}

Funding No funding was used to conduct this meta-analysis.

Conflicts of interest All authors de clare that they do not have any conflict of interest to declare.

Availability of data and material Data will be available with corresponding author and may be accessed on request.

Code availability Not applicable.

Ethics approval Not applicable.

Consent to participate Not applicable.

Consent for publication Not applicable.

\section{References}

1. Nutho B, Mahalapbutr P, Hengphasatporn K, Pattaranggoon NC, Simanon N, Shigeta Y, et al. Why are lopinavir and ritonavir effective against the newly emerged coronavirus 2019 ? Atomistic insights into the inhibitory mechanisms. Biochemistry. 2020;59:1769-79. https://doi.org/10.1021/acs.biochem.0c00160.

2. WHO Coronavirus disease (COVID-19): Situation Report-193. 2020. https://www.who.int/docs/default-source/coronaviruses/ situation-reports. Accessed 04 October 2021.

3. Chen N, Zhou M, Dong X, Qu J, Gong F, Han Y, et al. Epidemiological and clinical characteristics of 99 cases of 2019 novel coronavirus pneumonia in Wuhan, China: a descriptive study. Lancet. 2020;395:507-13. https://doi.org/10.1016/S0140-6736(20) 30211-7.

4. Huang C, Wang Y, Li X, Ren L, Zhao J, Hu Y, et al. Clinical features of patients infected with 2019 novel coronavirus in Wuhan, China. Lancet. 2020;395:497-506. https://doi.org/10.1016/S01406736(20)30183-5.

5. Rameshrad M, Ghafoori M, Mohammadpour AH, Nayeri MJD, Hosseinzadeh H. A comprehensive review on drug repositioning against coronavirus disease 2019 (COVID19). Naunyn Schmiedebergs Arch Pharmacol. 2020;393:1137-52. https://doi.org/10. 1007/s00210-020-01901-6.

6. Samuel CE. Antiviral actions of interferons. Clin Microbiol Rev. 2001;14:778-809.

7. Levy DE, García-Sastre A. The virus battles: IFN induction of the antiviral state and mechanisms of viral evasion. Cytokine Growth Factor Rev. 2001;12:143-56. https://doi.org/10.1016/ s1359-6101(00)00027-7.

8. Biron CA. Interferons alpha and beta as immune regulatorsa new look. Immunity. 2001;14:661-4. https://doi.org/10.1016/ s1074-7613(01)00154-6.

9. Lokugamage KG, Hage A, de Vries M, Valero-Jimenez AM, Schindewolf C, Dittmann M, et al. SARS-CoV-2 is sensitive to type I interferon pretreatment. bioRxiv [Preprint]. 2020. https:// doi.org/10.1101/2020.03.07.982264 ((update in: J Virol. 2020;94)).

10. Hung IF, Lung KC, Tso EY, Liu R, Chung TW, Chu MY, et al. Triple combination of interferon beta-1b, lopinavir-ritonavir, and ribavirin in the treatment of patients admitted to hospital with COVID-19: an open-label, randomised, phase 2 trial. Lancet. 2020;395:1695-704. https://doi.org/10.1016/S0140-6736(20) 31042-4.
11. Knoops K, Kikkert M, Worm SH, Zevenhoven-Dobbe JC, van der Meer Y, Koster AJ, et al. SARS-coronavirus replication is supported by a reticulovesicular network of modified endoplasmic reticulum. PLoS Biol. 2008;6: e226. https://doi.org/10.1371/journ al.pbio.0060226.

12. Snijder EJ, van der Meer Y, Zevenhoven-Dobbe J, Onderwater JJ, van der Meulen J, Koerten HK, et al. Ultrastructure and origin of membrane vesicles associated with the severe acute respiratory syndrome coronavirus replication complex. J Virol. 2006;80:5927-40. https://doi.org/10.1128/JVI.02501-05.

13. Siu KL, Yeung ML, Kok KH, Yuen KS, Kew C, Lui PY, et al. Middle east respiratory syndrome coronavirus 4a protein is a double-stranded RNA-binding protein that suppresses PACT-induced activation of RIG-I and MDA5 in the innate antiviral response. J Virol. 2014;88:4866-76. https://doi.org/10.1128/JVI.03649-13.

14. Frieman M, Ratia K, Johnston RE, Mesecar AD, Baric RS. Severe acute respiratory syndrome coronavirus papain-like protease ubiquitin-like domain and catalytic domain regulate antagonism of IRF3 and NF-kappaB signaling. J Virol. 2009;83:6689-705. https://doi.org/10.1128/JVI.02220-08.

15. Royal College of Physicians. National Early Warning Score (NEWS) 2. RCP, 2017. www.rcplondon.ac.uk/projects/outputs/ national-early-warning-score-news-2. Accessed 4 Aug 2021.

16. Monk PD, Marsden RJ, Tear VJ, Brookes J, Batten TN, Mankowski M, et al. Inhaled Interferon Beta COVID-19 Study Group. Safety and efficacy of inhaled nebulised interferon beta-1a (SNG001) for treatment of SARS-CoV-2 infection: a randomised, double-blind, placebo-controlled, phase 2 trial. Lancet Respir Med. 2021;9:196-206. https://doi.org/10.1016/S2213-2600(20) 30511-7.

17. Khamis F, Al Naabi H, Al Lawati A, Ambusaidi Z, Al Sharji M, Al Barwani U, et al. Randomized controlled open label trial on the use of favipiravir combined with inhaled interferon beta- $1 \mathrm{~b}$ in hospitalised patients with moderate-to-severe COVID-19 pneumonia. Int J Infect Dis. 2021;102:538-43. https://doi.org/10.1016/j. ijid.2020.11.008.

18. Rahmani H, Davoudi-Monfared E, Nourian A, Khalili H, Hajizadeh $\mathrm{N}$, Jalalabadi NZ, et al. Interferon $\beta-1 \mathrm{~b}$ in treatment of severe COVID-19: a randomized clinical trial. Int Immunopharmacol. 2020;88: 106903. https://doi.org/10.1016/j.intimp.2020.106903.

19. Davoudi-Monfared E, Rahmani H, Khalili H, Hajiabdolbaghi M, Salehi M, Abbasian L, et al. A randomized clinical trial of the efficacy and safety of interferon $\beta-1 \mathrm{a}$ in treatment of severe COVID19. Antimicrob Agents Chemother. 2020;64:e01061-e1120. https://doi.org/10.1128/AAC.01061-20.

20. Alavi Darazam I, Shokouhi S, Pourhoseingholi MA, Naghibi Irvani SS, Mokhtari M, Shabani M, et al. Role of interferon therapy in severe COVID-19: the COVIFERON randomized controlled trial. Sci Rep. 2021;11:8059. https://doi.org/10.1038/ s41598-021-86859-y.

21. WHO Solidarity Trial Consortium, Pan H, Peto R, HenaoRestrepo AM, Preziosi MP, Sathiyamoorthy V, Abdool Karim Q, et al. Repurposed antiviral drugs for Covid-19-interim WHO solidarity trial results. N Engl J Med. 2021;384:497-511. https:// doi.org/10.1056/NEJMoa2023184.

22. Alavi Darazam I, Hatami F, Mahdi Rabiei M, Amin Pourhoseingholi M, Shabani M, Shokouhi S, et al. An investigation into the beneficial effects of high-dose interferon beta 1-a, compared to low-dose interferon beta 1-a in severe COVID-19: the COVIFERON II randomized controlled trial. Int Immunopharmacol. 2021;99: 107916. https://doi.org/10.1016/j.intimp.2021.107916.

23. World Health Organization. WHO COVID-19 Solidarity Therapeutics Trial. https://www.who.int/emergencies/diseases/novelcoronavirus-2019/global-research-on-novel-coronavirus-2019ncov/solidarity-clinical-trial-for-covid-19-treatments. Accessed 22 Oct 2021. 
24. Adaptive COVID-19 treatment trial 3 (ACTT3). https://clinicaltr ials.gov/ct2/show/NCT04492475. Accessed 8 Aug 2021.

25. Nakhlband A, Fakhari A, Azizi H. Interferon-beta offers promising avenues to COVID-19 treatment: a systematic review and meta-analysis of clinical trial studies. Naunyn Schmiedebergs Arch Pharmacol. 2021;394:829-38. https://doi.org/10.1007/ s00210-021-02061-x.

26. Sosa JP, Ferreira Caceres MM, Ross Comptis J, Quiros J, Príncipe-Meneses FS, Riva-Moscoso A, et al. Effects of interferon beta in COVID-19 adult patients: systematic review. Infect Chemother. 2021;53:247-60. https://doi.org/10.3947/ic.2021.0028.

27. Kaidashev I, Shlykova O, Izmailova O, Torubara O, Yushchenko Y, Tyshkovska T, et al. Host gene variability and SARS-CoV-2 infection: a review article. Heliyon. 2021;7: e07863. https://doi. org/10.1016/j.heliyon.2021.e07863. 